\title{
Effect of detergents as antibacterial agents on biofilm of antibiotics-resistant Vibrio parahaemolyticus isolates
}

\begin{abstract}
Vibrio parahaemolyticus (V. parahaemolyticus) is a halophilic, Gram-negative human pathogen known as a leading cause of seafood-derived food poisoning. Due to high contamination rate of seafood in Asian countries, V. parahaemolyticus is considered as a food safety concern. V. parahaemolyticus is able to produce biofilm which is more resistant toward disinfectants and antibodies than its planktonic form. Thirty six V. parahaemolyticus isolates from seafood were tested for their susceptibility using 18 different antibiotics. Two V. parahaemolyticus isolates were resistant to bacitracin, chloramphenicol, rifampin, ampicillin, vancomycin, nalidixic acid, penicillin and spectinomycin. Fourteen V. parahaemolyticus isolates were found to be resistant to bacitracin, tetracycline, rifampin, ampicillin, vancomycin, penicillin and spectinomycin. The remaining two isolates were resistant to more than 2 antibiotics. Majority of the V. parahaemolyticus isolates $(97.2 \%)$ showed MAR index > 0.2, indicating that these isolates were originated from high risk sources. To investigate effect of three common detergents on antibacterial-resistant $\mathrm{V}$. parahaemolyticus, $16 \mathrm{~V}$. parahaemolyticus isolates resistant to more than 7 antibiotics were selected. V. parahaemolyticus (ATCC 17802) was used as reference strain. Detergents were tested for their minimum inhibitory concentration (MIC) and minimal bactericidal concentration (MBC) and time-kill curves were constructed to assess the concentration between MIC and bactericidal activity of detergents. Detergents D1 (Linear alkyl benzene based) was found to be the most effective with MIC and MBC ranged between 97.656 and $1562.5 \mu \mathrm{g} / \mathrm{ml}$ and $781.25-3125 \mu \mathrm{g} / \mathrm{ml}$, respectively. The time-kill curves demonstrated that the bactericidal endpoint for resistant V. parahaemolyticus isolates reached after 30 min incubation with D1 at concentration $8 \times$ MIC. The isolate VP003 was killed at $8 \times$ MIC within $0.5 \mathrm{~h}$ and the reduction in $\mathrm{CFU} / \mathrm{ml}$ was $3 \mathrm{log}$ units $(99.9 \%)$. V. parahaemolyticus biofilms were formed in 96 wells microtiter plates at $37^{\circ} \mathrm{C}$ and $24 \mathrm{~h}$-old biofilm were used to test antibacterial activity of detergents. Results showed that biofilm-producing ability of antibacterial-resistant V. parahaemolyticus isolates were inhibited at $1562.5-6250 \mu \mathrm{g} / \mathrm{ml}$ of $\mathrm{D} 1$ and eradicated at $3125-\geq 50,000 \mu \mathrm{g} / \mathrm{ml}$ of D1. Detergents showed potential antimicrobial activity against $\mathrm{V}$. parahaemolyticus.
\end{abstract}

Keyword: Antibacterial; Vibrio parahaemolyticus; Seafood; Biofilm 